treatment was documented. We used the MedCalc statistical software for statistical analysis.

Results We identified 124 infants (66 males and 58 females). Of these $43(34.7 \%)$ were diagnosed with EO-NS and $81(65.3 \%)$ with LO-NS. While the rate of positive blood culture results was $36 \%$ in the EO-NS group it was $84 \%$ in the LO-NS group. Treatment success rates were $93,5 \%$ in the EO-NS group, and $81,7 \%$ in the LO-NS group. Carbapenem resistance was identified in $10,5 \%$ of the newborns in the LO-NS group. Mortality rates were $4.6 \%$ in the EO-NS and $8,9 \%$ in the LO-NS group.

Conclusions The success rates of empiric antibiotic treatment was high in both the EO-NS and LO-NS group. The microbial flora of the NICU should be taken into account when deciding for the empirical antibiotic treatment regimens in infants with hospital acquired sepsis.

\section{PO-0572 UMBILICAL VENOUS CATHETERS WITH AGION ANTIMICROBIAL SYSTEM IN A DUTCH NICU}

A van den Hoogen. Neonatology, University Medical Center Utrecht, Utrecht, Netherlands 10.1136/archdischild-2014-307384.1213

Background The majority of preterm infants at NICUs receives a central venous, or umbilical vein catheter (UVC) and is therefore at risk for catheter associated sepsis. Silver-impregnated UVCs with the $\mathrm{AgION}^{\mathrm{TM}}$ antimicrobial system may prevent sepsis and may have longer insertion time.

Objective To assess sepsis and additional CVC insertion with the use of silver-impregnated UVCs compared with conventional ones.

Methods Catheter-duration, sepsis and additional CVC use was compared between infants with silver-impregnated UVCs (silvergroup) during 1 year (2012-2013) and infants with conventional UVCs (controls) during 2011, when inserted $>3$ days.

Results In 156/249 (2012-2013) infants a silver-impregnated and all 273 with an UVC in 2011 a conventional UVC was inserted. Mean catheter-duration was 5.8 (3-15) days in the silvergroup vs 5.7 (3-12) days in the controls (NS). 11/156 (7\%) infants from the silvergroup developed sepsis during catheterisation vs 17/267 (6.4\%) controls [NS]. Main causative microorganisms: CoNS (62.5\%), S. aureus (15.6\%), Enterobacter (9.3\%). In $22 / 156$ infants of the silvergroup, UVC use was longer than 8 days, vs in 20/273 controls (NS). 3/22 of the silvergroup with UVC use $>8$ days developed sepsis vs $1 / 273$ controls ( $p=$ $0.015)$. Significant more infants in the silvergroup needed additional CVC insertion $18 / 156(11.5 \%)$ vs in $28 / 273(10.3 \%)$ controls $(\mathrm{p}=0.00)$.

Conclusions Duration of $>8$ days of silver-impregnated UVC significantly increased the risk for sepsis as compared with conventional UVC use.

Silver-impregnated UVCs were not inserted for longer periods than conventional UVCs.

Anti-infectious advantage of the silver-impregnated UVCs could not be proven.

\section{PO-0573 A TALE OF TWO CRP'S; IMPLEMENTING THE NICE EARLY ONSET SEPSIS GUIDELINE}

M Winton, SBilal, S Douglass, C Beaves, K Psychogiou, LIZ McKechnie. Neonates, Leeds Teaching Hospital NHS Trust, Leeds West Yorkshire, UK
Introduction A rational approach to managing babies at risk of early onset sepsis continues to challenge neonatal units. In August 2012 NICE published guidance on antibiotics for early onset sepsis in neonates (1). We review our unit's performance in implementing the NICE guidance. Baseline assessment using the NICE tool had been completed previously and the NICE guideline implemented with adjustments for local use.

Method A three month prospective audit of babies at risk of or suspected of having early onset sepsis (sepsis within $72 \mathrm{~h}$ of birth). The NICE guideline audit tool was used. (3).

Results 64 babies were audited. Every baby had a blood culture taken before commencement of antibiotics and were started on correct antibiotic doses. Initial CRP's were taken in 95\% of cases but only $61 \%$ had a repeat at $24 \mathrm{~h}$.

$69 \%$ of babies received antibiotics within $1 \mathrm{~h}$ of making clinical decision. $88 \%$ had cultures available at $48 \mathrm{~h}$ as per local policy.

Discussion Our data demonstrates the challenge of implementing a relatively straight forward protocol of care. We excelled in some elements: initial investigation and prescribing accuracy. There was clear room for improvement in other areas.

Simple changes to practice have subsequently been implemented including revised gentamicin prescription charts and education to highlight the importance of timely administration of antibiotics and the evidence behind checking CRP levels (5, 6, 7). Re-audit is planned for early 2014 .

\section{PO-0574 INDICATIONS AND OUTCOMES OF LUMBAR PUNCTURES IN TERM NEONATES IN A TERTIARY NEONATAL UNIT}

H Wood, E Seager, J Gray, SV Rasiah. Neonatology, Birmingham Women's NHS Foundation Trust, Birmingham, UK

10.1136/archdischild-2014-307384.1215

Background Lumbar puncture (LP) is usually performed when there is a clinical suspicion of meningitis in babies with suspected sepsis. NICE recently published their guidelines on 'antibiotics for early-onset neonatal infections' with guidance on when LPs should be considered.

Aim To audit the indications and outcomes of LPs performed in term babies $(>37+0$ weeks) in a tertiary neonatal unit.

Methods A list of term babies who had an LP was obtained from the Microbiology Department between 01/01/2010 and $31 / 12 / 2013$. The Badger electronic patient record and hospital blood results systems were reviewed to collect the data.

Results In the last 4 years we had 2,882 term babies admitted to the neonatal unit. 136 LPs were performed in 133 term babies. The reasons for LPs were; (a) raised CRP in 106 cases (median CRP was 70), (b) abnormal neurology in 18 cases, (c) positive blood culture in 8 cases and (d) 4 were for no other clinical focus. There was one culture of coliforms and another positive for herpes simplex virus type 1 on PCR. At discharge, 8 had a diagnosis of meningitis and 1 with encephalitis.

Conclusion The predominant indication for LPs in term babies was a raised CRP. We only isolated organisms from two samples. As per NICE guidance, we rely on a combination of clinical findings and CRPs when deciding which term babies to LP.

\section{REFERENCES}

Antibiotics for early-onset neonatal infection - NICE www.nice.org.uk/nicemedia/live/ 13867/60633/60633.pdf? 